\author{
Christopher M. Powers, ${ }^{1}$ Ph.D., P.T.; Mark G. Blanchette, ${ }^{1}$ M.S.; John R. Brault, ${ }^{2}$ M.S.; \\ Jim Flynn, ${ }^{3}$ P.E.; and Gunter P. Siegmund, ${ }^{4}$ Ph.D., P.Eng.
}

\title{
Validation of Walkway Tribometers: Establishing a Reference Standard*
}

\begin{abstract}
Tribometers are mechanical devices used to measure walkway coefficient of friction (COF) for the purpose of assessing slip risk. The purpose of this study was to define a tribometer reference standard and use it to assess the performance of various tribometers. Eighty subjects were randomly assigned to walk across one of four wet walkway surfaces (polished black granite, porcelain, vinyl composition tile, and ceramic tile) to establish the relative slipperiness of each surface. Eleven tribometers were subsequently used to measure and rank the COF of all four surfaces. Our results revealed that only four of the 11 tribometers (Wessex pendulum, Sigler pendulum, Mark II, and Mark III) met our compliance criteria by both correctly ranking all four surfaces and differentiating between surfaces of varying degrees of slipperiness. Our protocol demonstrates that human gait-based measures of slipperiness can be used to create reference standards against which tribometer measurements can be validated.
\end{abstract}

KEYWORDS: forensic science, walkway safety, walkway tribometer, slips, coefficient of friction, reference standard

Tribometers are mechanical devices used by the safety community to measure walkway surface coefficient of friction (COF) for the purpose of assessing the risk of slipping. Tribometers also are used by the flooring and footwear industries to assess product performance. Currently, over 30 different tribometer models using a wide range of mechanical designs and COF calculation methods are being used by these various industries (1-3). To date, no tribometer model or mechanical test to measure COF relevant to human ambulation has been universally accepted. The fact that the measurement of friction is a function of both the material being tested and the measuring system itself explains why several studies have shown that different devices yield different $\mathrm{COF}$ measurements for the same surface (4-15). For example, a study of nine tribometers demonstrated an extremely wide range of COF values for the same surface when tested under wet conditions (0.06-0.69) (14).

Inconsistent measurement is one of the reasons why every measurement device should have a reference standard against which to validate and calibrate its performance; however, no such reference standard exists for tribometers. The consensus standards organizations that oversee footwear and walkway safety have recently recognized the importance of developing a protocol for validating tribometers to verify their ability to assess the safety of footwear, floor products, and walkway surfaces $(16,17)$.

\footnotetext{
${ }^{1}$ Musculoskeletal Biomechanics Research Laboratory, Division of Biokinesiology \& Physical Therapy, University of Southern California, 1540 E. Alcazar St., CHP-155, Los Angeles, CA 90089.

${ }^{2}$ Semper Scientific, 26021 Acero, Mission Viejo, CA 92691.

${ }^{3}$ J2 Engineering Inc., 7636 N. Ingram Ave, Suite 108, Fresno, CA 93711.

${ }^{4}$ MEA Forensic Engineers \& Scientists, 11-11151 Horseshoe Way, Richmond, BC, Canada V7A 4S5.

*Presented at the North American Conference on Biomechanics, August 6, 2008, in Ann Arbor, MI

Received 23 Sept. 2008; and in revised form 15 Jan. 2009; accepted 9 Mar. 2009.
}

In a previous publication, we introduced the concept of using human subject slip risk to create a reference standard against which tribometer measurements could be validated (14). In this prior study, human subjects $(N=84)$ walked across three different surfaces with and without a contaminant (six conditions). The number of slips recorded on each surface was used to establish the relative slipperiness of each surface condition. We then proposed two objective criteria to establish the validity of a given tribometer. First, a tribometer had to correctly rank the COF of the different surfaces in the order of slip risk as determined by the human subject walking trials. Second, the COF of the surfaces of varying slipperiness had to be statistically different from each other. Results revealed that only two of the nine tribometers tested (Tortus II and the Mark III) met the compliance criteria by both correctly ranking all six conditions and differentiating between surfaces of differing degrees of slipperiness. However, a criticism of this study was that two of the three surfaces were not standard walkway surfaces. Establishing a reference standard using atypical materials could preclude its use for the validation of tribometers normally used to measure the COF of flooring materials commonly encountered by pedestrians. A second limitation of our previous study was that both wet and dry surface conditions were used. Typically, slips do not occur under dry conditions. Using a protocol similar to our previous study (14), the current investigation sought to address the aforementioned limitations and define a walkway tribometer reference standard and assess its use by various tribometers.

\section{Methods}

\section{Human Subject Testing}

Subjects-Eighty subjects (23 males, 57 females) between the ages of 20 and 39 years (mean age $26.3 \pm 4.8$ years) were recruited 
for this portion of the study. All subjects were healthy and capable of independent ambulation. Subjects who reported any orthopedic injury, medical condition, or pregnancy were excluded from participation. Prior to testing, each subject signed an informed consent approved by the Institutional Review Board of the University of Southern California.

Floor Surfaces and Conditions-Four flat smooth surfaces were tested: polished black granite, porcelain, ceramic tile, and vinyl composition tile (VCT). Each surface consisted of a $0.6 \times 1.2 \mathrm{~m}$ rectangle embedded in the center of a $10-\mathrm{m}$ walkway. A sufficient amount of distilled water was applied to each surface to create a continuous film. For the black granite surface, Triton X100 nonionic surfactant (Gallade Chemical, Santa Ana, CA) was mixed into the water $(200-\mu \mathrm{L} / 500-\mathrm{mL})$ to avoid beading and improve wetting.

Procedures-All testing (tribometer and human subject) was performed at the Musculoskeletal Biomechanics Research Laboratory at the University of Southern California. The temperature and humidity in the laboratory during testing were $70^{\circ} \mathrm{F}$ and $34 \%$, respectively. To rank the slipperiness of the different surfaces, subjects were randomly assigned to walk across one of the four floor surfaces (20 subjects per group). Several studies have shown that awareness of a potential slip condition and prior slip experience can lead to alterations in gait (18-20). Therefore, to limit gait adjustments that may have affected the slip trial outcome, subjects only were exposed to one surface. The four groups were similar in terms of age, height, and weight (Table 1).

To control for the influence of footwear, subjects were provided with a pair of Oxford-style shoes in their size. The soles of these shoes consisted of a smooth styrene butadiene rubber with a 75 Shore A hardness (Quabaug Corp., North Brookfield, MA). The soles represented the most common shoe bottom material used globally in the year 2001 (William Ells, Quabaug Corp., pers. comm.). Before each test session, the floor was swept for dust and both the floor panel and shoe soles were cleaned with $70 \%$ isopropyl alcohol.

During all walking trials, subjects were required to wear a fallarresting harness attached to an overhead low-friction trolley that extended the length of the walkway. Subjects first performed several walking trials on a dry, nonslippery floor condition. These trials were used to adjust the subjects' starting position to ensure a clean foot strike on the target surface. The mean walking velocity for all trials was $2.09 \pm 0.15-\mathrm{m} / \mathrm{sec}$ as determined via photoelectric triggers placed at both ends of the walkway.

To reduce awareness of which trial contained the test surface, subjects left the room for a similar period of time between each trial (c. $2 \mathrm{~min}$ ). Subjects also wore goggles with the lower half blacked out and were instructed to look at a spot on the far wall as

TABLE 1-Subject characteristics mean (SD).

\begin{tabular}{|c|c|c|c|c|}
\hline & $\begin{array}{l}\text { Black } \\
\text { Granite }\end{array}$ & Porcelain & Ceramic & $\begin{array}{c}\text { Vinyl } \\
\text { Composition } \\
\text { Tile }\end{array}$ \\
\hline & $N=20$ & $N=20$ & $N=20$ & $N=20$ \\
\hline Females/males & $16 / 4$ & $15 / 5$ & $14 / 6$ & $12 / 8$ \\
\hline Age (years) & $28.3(5.7)$ & $26.0(5.2)$ & $25.4(3.7)$ & $25.7(4.1)$ \\
\hline Height $(\mathrm{cm})$ & $167.8(11.0)$ & $165.0(9.5)$ & $169.3(7.9)$ & $170.8(7.3)$ \\
\hline Weight (kg) & $63.5(13.7)$ & $62.5(12.2)$ & 67.5 (13.9) & $70.6(13.8)$ \\
\hline $\begin{array}{l}\text { Walking speed } \\
(\mathrm{m} / \mathrm{sec})\end{array}$ & $2.05(0.16)$ & $2.15(0.15)$ & $2.05(0.12)$ & $2.12(0.17)$ \\
\hline
\end{tabular}

they traversed the walkway. Lighting was dimmed to minimize reflections from the wet surfaces, and a "spotter" at the far end of the laboratory gave the appearance that the test surface was near the end rather than the middle of the walkway. To eliminate the effect of prior experience, subjects were exposed to their assigned test surface for only one trial.

Slip Definition During Walking-To confirm whether a slip occurred on a given surface, an 8-camera motion analysis system (Vicon; Oxford Metric Ltd., Oxford, U.K.) was used to capture human subject motion data at $120-\mathrm{Hz}$. Reflective markers were placed on the heel and second metatarsal head area on subjects' shoes. Heel slips were defined as a minimum $4 \mathrm{~cm}$ anterior displacement of the heel marker following the initial contact phase of gait (21). Toe slips were defined as any negative (posterior-directed) velocity of the toe marker before toe-off phase of gait (14). In all cases, digital video was used to confirm the presence of a slip.

\section{Tribometer Testing}

Tribometers-Eleven tribometers were used to measure the COF of the four surfaces (Table 2). One tribometer (BOT 3000) was tested using static and dynamic modes. Each tribometer was operated by an experienced user of that device, and testing followed protocols provided by the manufacturer or as set forth in applicable standards from ASTM International (West Conshohocken, PA). A second individual recorded the tribometer results, while a third individual monitored the testing protocol to ensure consistent technique and correct recording. The value measured by each tribometer was assumed to represent the COF measured by that tribometer unless the manufacturer specified the required use of a conversion factor.

Procedures-COF testing with the tribometers was performed on the four floor surfaces under the same laboratory conditions present at the time of the walking trials. The tribometer test order for measuring the four surfaces was randomly designated. The surface wetting protocol was the same as that used for the human subject portion of the study. Prior to testing, the test foot of each tribometer was cleaned and prepared according to manufacturer specifications. This procedure was repeated prior to the testing of each surface. The independent observer ensured that a continuous wet film was present on the test surface. The COF of each surface was measured four times, once in each orthogonal direction (i.e., $0^{\circ}, 90^{\circ}, 180^{\circ}$, and $270^{\circ}$ ) relative to the longitudinal axis of the walkway.

Data Analysis-Each human subject walking trial was classified as a no slip, toe slip, or heel slip. To test for differences in the type of slip that occurred on the four surfaces, a chi-squared test for homogeneity was performed on a $4 \times 3$ (floor surface $\times$ slip type) contingency table at a significance level of $\alpha=0.05$.

To test for differences between the measured $\mathrm{COF}$ values for each surface, the mean and standard deviations of the COF values for each tribometer/surface combination were first calculated. For each tribometer, a one-way analysis of variance (ANOVA) was used to determine whether significant differences were present between the four surfaces. Post hoc tests were conducted using a Fischer least significant difference. The significance level for each ANOVA was $\alpha=0.05$ and was not adjusted for multiple comparisons because the number of tribometers chosen for the study should not influence whether or not a specific tribometer identified the slipperiness of a specific pair of surfaces as significantly different. 
TABLE 2-Tribometer characteristics: tribometer name, type, and test foot material.

\begin{tabular}{|c|c|c|}
\hline Device & Operating Principle & Test Foot (Material/Size) \\
\hline English XL* & Inclinable mast (pneumatic driven) & Neolite $^{\circledR}$ (13 mm diameter) \\
\hline BOT $3000^{\dagger}$ & Drag sled-motor driven & Leather $(27.9 \times 27.9 \mathrm{~mm})$ \\
\hline $\mathrm{C} 1028^{*}$ & Drag sled-manually pulled & Neolite $^{\circledR}(76 \times 76 \mathrm{~mm})$ \\
\hline Tortus $\mathrm{II}^{\S}$ & Drag sled-motor driven & $4 \mathrm{~S}$ rubber ( $9.5 \mathrm{~mm}$ diameter $)$ \\
\hline Tortus III ${ }^{\S}$ & Drag sled-motor driven & $4 \mathrm{~S}$ rubber ( $9 \mathrm{~mm}$ diameter) \\
\hline Horizontal Pull Slipmeter & Drag sled-motor pulled & Neolite $\left.^{(}\right)$(13 mm diameter) \\
\hline Wessex Pendulum** & Pendulum & $4 \mathrm{~S}$ rubber $(76 \times 2 \mathrm{~mm})$ \\
\hline Sigler Pendulum ${ }^{\dagger \dagger}$ & Pendulum & Neolite $^{(8)}(38 \times 38 \mathrm{~mm})$ \\
\hline Mark I & Inclinable mast (gravity driven) & Neolite $^{\circledR}(76 \times 76 \mathrm{~mm} ;$ nongrooved $)$ \\
\hline Mark II ${ }^{\S \S}$ & Inclinable mast (gravity driven) & Neolite $^{(\circledR)}(76 \times 76 \mathrm{~mm} ;$ grooved $)$ \\
\hline Mark III ${ }^{\S \S}$ & Inclinable mast (spring activated) & Neolite $^{(}(76 \times 76$ mm; grooved $)$ \\
\hline
\end{tabular}

*William English Inc., Alva, FL.

${ }^{\dagger}$ Regan Scientific Instruments, Southlake, TX.

${ }^{\star}$ Smithers Scientific Services, Akron, OH.

${ }^{\S}$ Severn Science Limited, Bristol, U.K.

Developed by Irvine/Liberty Mutual, no longer manufactured.

**Wessex Engineering Ltd., U.K.

${ }^{\dagger}$ Developed by Percy Sigler and National Bureau of Standards, no current manufacturer.

No specific manufacturer.

${ }^{\S}$ Slip-Test, Spring Lake, NJ.

Comparison of Human Subject and Tribometer Ranking-As reported previously (14), the slipperiness ranking determined from the walking trials was considered the reference against which the tribometer-determined ranking was compared. The tribometer measurements were compared to the gait-based ranking using two criteria. Criterion \#1: did a given tribometer rank the COF of the different surfaces in the same order indicated by the human subject results? Criterion \#2: did a given tribometer statistically differentiate between surfaces with significantly different levels of slip risk?

\section{Results}

The results of the human subject walking trials are presented in Table 3. All four surface conditions were significantly different from each other with respect to levels of slipperiness $(p<0.05)$. Black granite had the largest number of slips $(N=19)$, the majority of which were heel slips $(N=13)$. Porcelain produced a mixture of heel slips $(N=4)$, toe slips $(N=11)$, and no slips $(N=5)$. VCT induced several toe slips $(N=7)$ but no heel slips. Ceramic tile did not produce any slips. Using these results, the surfaces were characterized as most slippery (black granite), moderately slippery (porcelain), less slippery (VCT), and least slippery (ceramic tile).

The distinction between the most slippery surface containing primarily heel slips and the moderately slippery surface containing primarily toe slips was based on the premise that toe slips are not hazardous for the walker because of where they occur in the gait cycle. Toe slips take place in late stance as weight is being transferred off the slipping limb to the support limb. In contrast, heel slips occur in early stance when weight is being transferred onto the slipping limb which increases the risk of a fall compared to a toe slip (14).

TABLE 3-Slip results: walking trials.

\begin{tabular}{lcccc}
\hline Surfaces & No Slips & Toe Slips & Heel Slips & Ranking \\
\hline Black granite & 1 & 6 & 13 & Most slippery \\
Porcelain & 5 & 11 & 4 & Moderately slippery \\
VCT & 13 & 7 & 0 & Less slippery \\
Ceramic & 20 & 0 & 0 & Least slippery \\
\hline
\end{tabular}

VCT, Vinyl composition tile.
Across all tribometers and surfaces, COF measurements varied from a low of $0.05 \pm 0.01$ for the Wessex pendulum on black granite to a high of $0.94 \pm 0.03$ for the BOT 3000 (static mode) on ceramic tile. Within each surface, the most consistent range of $\mathrm{COF}$ values was observed on black granite (0.05-0.35) whereas the most varied range of $\mathrm{COF}$ values was observed on ceramic (0.24-0.94). Although all 11 tribometers were able to statistically differentiate levels of slipperiness between some of the surfaces, only six tribometers (Tortus III, Horizontal Pull Slipmeter [HPS], Wessex, Sigler, Mark II, and Mark III) were able to significantly differentiate all four surfaces (Criterion \#2 in Table 4).

A comparison of the tribometer COF measurements to the gait-based ranking of surface slipperiness revealed that only four tribometers (Wessex, Sigler, Mark II, and Mark III) satisfied our validation criteria by both ranking the surfaces in the correct order (Criterion \#1) and statistically differentiating the differing degrees of slipperiness between each of the four surfaces (Criterion \#2). The remaining seven tribometers failed Criterion \#1, Criterion \#2, or both.

Two tribometers (BOT 3000 and Mark I) were able to rank the surfaces in the correct order of slipperiness but failed to statistically differentiate among them (Table 4). The BOT 3000 (static mode) was unable to differentiate between VCT and ceramic tile. In dynamic mode, the BOT 3000 could not statistically differentiate black granite and porcelain. The Mark I could not differentiate between porcelain and VCT. Two tribometers (Tortus III and HPS) passed Criterion \#2 but failed Criterion \#1 (Table 4). Both tribometers failed Criterion \#1 by incorrectly ranking ceramic as being more slippery than VCT.

Three tribometers (English XL, C1028, and Tortus II) failed both validation criteria (Table 4). The English XL incorrectly ranked VCT as more slippery than porcelain thereby failing Criterion \#1. Additionally, the English XL did not differentiate between these surfaces, thus failing Criterion \#2. The C1028 incorrectly ranked ceramic tile as more slippery than VCT and also failed to differentiate between the two surfaces thereby failing both validation criteria. The Tortus II failed Criterion \#1 by incorrectly ranking ceramic tile as more slippery than VCT. The Tortus II failed Criterion \#2 by not differentiating between ceramic tile and VCT. 


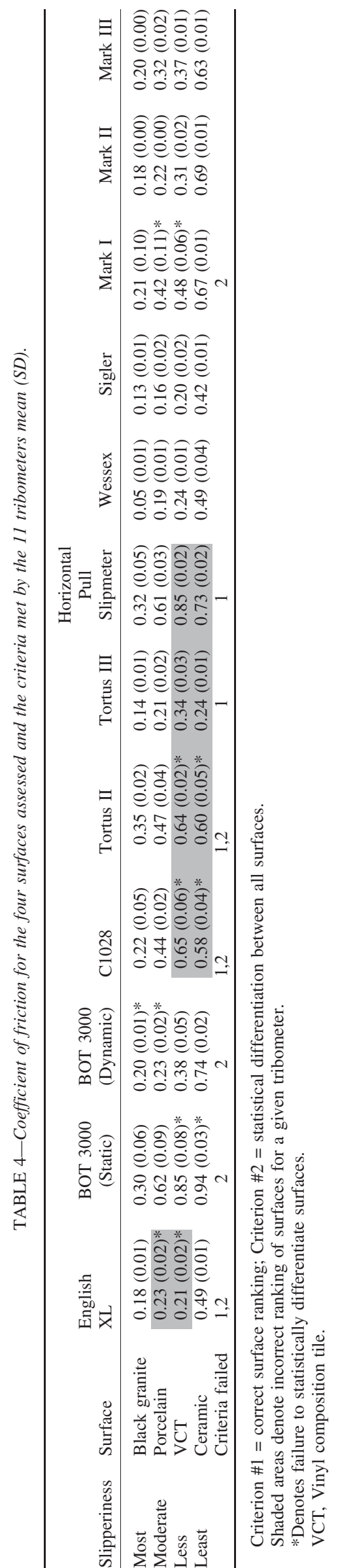

\section{Discussion}

The results of our tribometer testing are consistent with prior studies that have demonstrated that different tribometers give different COF values for the same surface (4-15). In the current study, wet porcelain was categorized as "moderately slippery" as 15 of 20 subjects slipped when walking across it; yet the range of COF measurements from the 11 tribometers spanned from 0.19 to 0.62 for this surface. This variability represents a problem to those in the walkway safety community who ascribe to the common approach of relating tribometer $\mathrm{COF}$ measurements to the required minimum (i.e., 0.21-0.26) for walking speeds in the range of $0.97-2.17-\mathrm{m} / \mathrm{sec}(22)$. As a result, the user of the tribometer that measured the wet porcelain COF as 0.19 would likely categorize the surface as very slippery because the surface supplies less friction than the pedestrian demand. The user of the tribometer that measured a COF of 0.62 might categorize that same surface as slip resistant as the measured COF far exceeds the required pedestrian demand. The wide range of tribometer $\mathrm{COF}$ readings underscores not only the need for a tribometer reference standard to be used for validation and calibration, but also the difficulty in ascribing a single safe threshold $\mathrm{COF}$ to a given floor surface to indicate the floor's potential for causing a slip.

The ability of the four surfaces used in the current study to statistically differentiate human subject slip risk on a continuum from most to least slippery demonstrates that this suite of materials can provide a means by which tribometer measurements can be compared and validated. Even after the criticisms directed at our earlier study were used in the design of the present investigation, very few of the tribometers tested produced acceptable results. Only four tribometers (Wessex, Sigler, Mark II, and Mark III) met our compliance criteria by correctly ranking all four surface conditions and differentiating between surfaces of differing degrees of slipperiness as established by the human subject walking trials.

The tribometers that passed our compliance criteria represent two different operating principles. The Sigler and Wessex are pendulum devices that measure the energy loss of a swinging test foot to derive COF values. The Mark II and Mark III are inclinable-mast tribometers designed such that the test foot strikes the surface at a known angle and speed. The devices are of similar design with the main difference being that the Mark II is gravity driven whereas the Mark III is spring activated. In addition, one distinguishing characteristic of the Mark II and Mark III is that they use grooved test feet (as recommended by the manufacturer). The grooves cut into the test foot (c. $3 \mathrm{~mm}$ deep by $1.5 \mathrm{~mm}$ wide and spaced $5 \mathrm{~mm}$ apart) may better replicate the dynamics of a shoe heel with a tread pattern landing on a wet walkway surface compared to a smooth test foot. Additionally, a previous study has shown that the Mark II better approximates the peak impact force underfoot early in the gait cycle when slips are most likely to occur (15).

Contrary to our previous testing (14), the Tortus II did not pass either criterion in the current study. This motor-driven tribometer has a small test foot $(9.5-\mathrm{mm}$ diameter) that is held in contact with the surface under a fixed load as it slides across the surface. Other investigators have implicated the drag sleds' prolonged surface residence time as a cause of adhesion, leading to $\mathrm{COF}$ measurements on wet surfaces that are greater than or equal to slip resistant dry surfaces $(9,12,23)$. This adhesion may account for the poor results of the other drag sled tribometers evaluated in our study (BOT 3000, Tortus III, HPS, and C1028). 
As stated earlier, the statistically differentiated slip risk demonstrated by the four walkway surfaces used in the current study provides an objective foundation for the validation and calibration of tribometers. We believe that these four surfaces could define a reference standard that tribometer manufacturers could use to validate their devices by demonstrating that the instrument's COF measurements properly rank and statistically differentiate these surfaces. The tribometer supplier also could use the reference surfaces to establish reference COF values to be provided to tribometer users so that users could independently calibrate their instruments by verifying consistent results.

Because only one tribometer of each model was included in the current study, our results do not necessarily invalidate all the instruments of a particular model. For instance, while the English XL used in this study failed to pass both compliance criteria, subsequent testing of 15 English XL tribometers revealed that five passed both criteria (J. Flynn, unpublished data). This illustrates the importance of using a reference standard to assess the validity and accuracy of each individual instrument.

\section{Summary}

The results of the current study are consistent with previous studies in that different tribometers yield different $\mathrm{COF}$ values for a given surface. Our results reinforce the need for objective criteria to ascertain which tribometers accurately evaluate floor slipperiness and a pedestrian's risk of slipping. Our protocol demonstrates that human gait-based measures of slipperiness can be used to create reference standards against which tribometer measurements can be validated. Given that only four of 11 tribometers passed our validation criteria, care should be taken in the interpretation of tribometer measurements in determining the safety of various walkway surfaces.

\section{References}

1. Chang WR, Gronqvist R, Leclercq S, Brungraber RJ, Mattke U, Strandberg $\mathrm{L}$, et al. The role of friction in the measurement of slipperiness, Part 2: survey of friction measurement devices. Ergonomics 2001; 44(13):1233-61.

2. Gronqvist R, Hirvonen M, Rajamaki E. Development of a portable test device for assessing on-site floor slipperiness: an interim report. Appl Ergon 2001;32(2): 163-71.

3. Skiba R, Scheil M, Windhovel U. Comparative research of unsteady measurements of friction coefficient on floors. Kaut Gummi Kunstst 1994;47:513-8.

4. Chang WR. The effect of surface roughness on the measurement of slip resistance. Int J Ind Ergon 1999;24:299-313.

5. Chang WR. The effects of slip criterion and time on friction measurements. Saf Sci 2002;40:593-611.

6. Chang WR, Matz S. The slip resistance of common footwear materials measured with two slipmeters. Appl Ergon 2001;32(6):549-58.

7. Dravitzki VK, Potter SM. The use of the tortus and the pendulum with the $4 \mathrm{~S}$ rubber for the assessment of slip resistance in the laboratory and the field. J Test Eval 1997;25(1):127-34.
8. Gronqvist R, Hirvonen M, Tohv A. Evaluation of three portable floor slipperiness testers. Int J Ind Ergon 1999;25:85-95.

9. Kulakowski BT, Buczek FL, Cavanagh PR, Pradhan P. Evaluation of performance of three slip resistance testers. J Test Eval 1989;17(4):234-40.

10. Marletta W. The effects of wetness on coefficient of friction measurement evaluated with three slip testers with varied residence times on selected materials. In: Proceedings of the Fourteenth Southern Biomedical Engineering Conference; 1995 April 7-9; Shreveport, LA. Piscataway, NJ: Institute of Electrical and Electronics Engineers Inc, 1995;167-70.

11. Marpet MI. Comparison of walkway-safety tribometers. J Test Eval 1996;25(9):245-54

12. Marpet MI. Comparison of walkway safety tribometers: part two. J Test Eval 1997;25(1):115-26.

13. Medoff H, Fleisher DH, Di Pilla S. Comparison of slip resistance measurements between two tribometers using smooth and grooved neolitestest-liner test feet. In: Marpet MI, Sapienza MA, editors. Metrology of pedestrian locomotion and slip resistance, ASTM STP 1424. West Conshohocken, PA: ASTM, 2002;67-72.

14. Powers CM, Brault JR, Stefanou MA, Tsai YJ, Flynn J, Siegmund GP. Assessment of walkway tribometer readings in evaluating slip resistance: a gait-based approach. J Forensic Sci 2007;52(2):400-5.

15. Powers CM, Kulig K, James F, Brault JR. Repeatability and bias of two walkway safety tribometers. J Test Eval 1999;27(6):368-74.

16. Hanson JP, Redfern MS, Mazumdar M. Predicting slips and falls considering required and available friction. Ergonomics 1999;42(12):161933.

17. Marpet MI. Issues in the development of modern walkway safety standards: required friction contextualization of test results, and non proprietary standards. In: Marpet MI, Sapienza MA, editors. Metrology of pedestrian locomotion and slip resistance. West Conshohocken, PA: ASTM, 2002;96-111.

18. Cham R, Redfern MS. Changes in gait when anticipating slippery floors. Gait Posture 2002;15(2):159-71.

19. Heiden TL, Sanderson DJ, Inglis JT, Siegmund GP. Adaptations to normal human gait on potentially slippery surfaces: the effects of awareness and prior slip experience. Gait Posture 2006;24(2):237-46.

20. Marigold DS, Patla AE. Strategies for dynamic stability during locomotion on a slippery surface: effects of prior experience and knowledge. J Neurophysiol 2002;88(1):339-53.

21. Cham R, Redfern MS. Heel contact dynamics during slip events on level and inclined surfaces. Saf Sci 2002;40(7):559-76.

22. Powers CM, Burnfield JM, Lim P, Brault JM, Flynn JE. Utilized coefficient of friction during walking: static estimates exceed measured values. J Forensic Sci 2002;47(6):1-6.

23. Bowman R, Stautins CJ, Westgate P, Quick GW. Implications for the development of slip-resistance standards from rank comparisons of friction test results obtained using different walkway safety tribometers under various conditions. In: Marpet MI, Sapienza MA, editors. Metrology of pedestrian locomotion and slip resistance. West Conshohocken, PA: ASTM, 2002;1121-36.

Additional information and reprint requests:

Christopher M. Powers, Ph.D., P.T.

Co-Director, Musculoskeletal Biomechanics Research Laboratory

Division of Biokinesiology \& Physical Therapy

University of Southern California

1540 E. Alcazar St., CHP-155

Los Angeles, CA 90089-9006

E-mail: powers@usc.edu 